\title{
PROPAGACIÓN IN VITRO DE Stevia rebaudiana BERT. (Asteraceae-Eupatorieae) A TRAVÉS DE ORGANOGÉNESIS IN VITRO PROPAGATION OF Stevia rebaudiana BERT. (Asteraceae-Eupatorieae) VIA ORGANOGENESIS
}

\author{
Isidro E. Suárez¹, José A. Salgado² \\ Recibido para evaluación: Diciembre 19 de 2007 - Aceptado para publicación: Abril 1 de 2008
}

\begin{abstract}
RESUMEN
Secciones de entrenudos tomados de plantas de Stevia rebaudiana establecidas en condiciones in vitro fueron inducidos a formar callos en presencia de diferentes concentraciones de Bencilaminopurina (BAP) combinadas con varias cantidades de ácido naftalenacético (ANA) evaluándose su efecto sobre el porcentaje de inducción de callo, friabilidad y formación de órganos. Posteriormente, los callos inducidos fueron multiplicados y el efecto de cuatro combinaciones de ANA y BAP sobre el incremento de masa fresca evaluado. Finalmente, los tallos proliferados fueron transferidos sobre medio de regeneración suplementado con diferentes cantidades de BAP solo o en combinación con ANA con el fin de evaluar su efecto sobre la regeneración de brotes. Todos los tratamientos fueron distribuidos utilizando un diseño completamente al azar con un mínimo de 15 repeticiones por tratamiento. Los resultados demostraron que la presencia de ANA es necesaria y suficiente para la formación de callo. La regeneración de brotes vía organogénesis ocurre de forma independiente a la presencia de RCV en el medio. Los tratamientos aplicados no afectaron el incremento de masa fresca de callo como tampoco tuvieron ningún efecto sobre la regeneración de plantas a partir del callo proliferado. Los datos colectados permiten recomendar un suplemento combinado de $1.0 \mathrm{mg} \mathrm{L}^{-1} \mathrm{ANA}+4.0 \mathrm{mg} \mathrm{L}^{-1}$ BAP para obtener plantas de Stevia rebaudiana a través de organogénesis.
\end{abstract}

Palabras Clave: Stevia, organogénesis, callo, reguladores de crecimiento.

\begin{abstract}
Nodal sections from in vitro established Stevia rebaudiana plants were cultured on medium independently supplemented with several NAA/BAP combinations to evaluate their effect on callus induction, friability and organ formation. Callus fresh weight as a result of NAA/BAP supply was determined and finally, BAP

'Universidad de Córdoba, Departamento de Ingeniería Agronó. Carrera 6 No. 76 -103 Tel (4) 7908023 Email: isuarez@sinu.unicordoba.edu.co

${ }^{2}$ Universidad de Córdoba, Candidato a M.Sc. Biotecnología
\end{abstract}


and BAP/NAA supply on shoot regeneration was evaluated. Treatments were distributed using a complete randomized design with at least 15 replicates per treatment. ANA turned out to be necessary and sufficient to induce callus formation. Shoot formation occurred during callus induction independently of PGR supply. PGR in the medium had neither effect on fresh weight increase nor on plant regeneration from proliferated callus. A $1.0 \mathrm{mg} \mathrm{L}^{-1} \mathrm{ANA}+4.0 \mathrm{mg} \mathrm{L}^{-1} \mathrm{BAP}$ supply in the medium is recommended for Stevia rebaudiana plant regeneration vía organogénesis.

Keywords: Stevia, organogenesis, callus, growth regulators.

\section{INTRODUCCIÓN}

Stevia rebaudiana Bertoni es una planta perenne, semi-arbustiva perteneciente a la familia Asteraceae, originaria del noreste paraguayo, en los límites con Brasil, donde crece en estado silvestre (Taiariol, 1995; Megeji et al., 2005); sus hojas contienen edulcorantes, esteviósidos y rebaudiósidos, que se estima poseen un poder endulzante que varía de 100 a 400 veces mayor que la sacarosa (Bespalhok et al., 1993; Bespalhok y Hattori, 1997; Soto y Del Vall, 2002). Esta capacidad endulzante ha conducido a considerarla como una buena alternativa para reemplazar edulcorantes que han sido cuestionados frecuentemente por su carácter artificial y sus efectos nocivos para la salud humana en largo plazo, como la sacarina, el aspartame y al acesulfame-K, entre otros (Taiariol, 1995).

La reproducción sexual de Stevia presenta ciertas desventajas que pueden afectar de forma negativa la eficiencia del cultivo como son la alta heterogeneidad de las poblaciones resultantes de sus semillas, la baja eficiencia de la germinación debido al alto porcentaje de semillas estériles y la ineficiencia de la recolección de la semilla por la desuniformidad en la floración y la maduración de la misma. Esta situación ha propiciado el uso de semilla vegetativa para la siembra de cultivos comerciales, la cual no está exenta de problemas debido a las bajas tasas de multiplicación por estacas, lo que ha obligado a la utilización de técnicas de micropropagación para hacer mas eficiente el proceso de multiplicación (Taiariol, 1995; Cassacia y Alvarez, 2006; Suárez et al., 2006).

Las dificultades en la producción de semilla sexual y la preferencia por el uso de material vegetativo para siembra han limitado los programas de mejoramiento genético en esta especie, arriesgando su evolución y el escape a limitantes del cultivo por medio de la resistencia genética. El cultivo de tejidos in vitro vía organogénesis representa una buena alternativa para aumentar las tasas de multiplicación de vegetales y una herramienta para inducir cambios en la estructura genética (Schwarz y Beaty, 2006). Existen pocos reportes de la micropropagación a través de organogénesis de la especie en cuestión, por lo que el desarrollo de esta forma alternativa de propagación podría también utilizarse en un futuro para la generación de variantes que ampliarían su base genética. La realización del presente trabajo de investigación, tuvo como objetivo, propagar in vitro plantas de Stevia a través del proceso de organogénesis, propendiendo el desarrollo de un protocolo de regeneración; que pudiera constituirse como una solución para la generación de variabilidad genética en esta especie.

\section{MATERIALES Y MÉTODOS}

\section{Inducción y multiplicación de callo}

Explantes consistentes de segmentos de 
entrenudos de aproximadamente $1.5 \mathrm{~cm}$ de longitud sin meristemos y obtenidos de plantas establecidas in vitro, fueron establecidos sobre medio de cultivo semisólido de Murashige y Skoog (1962) diluido a la mitad (1/2MS) suplementado con (en $\mathrm{mg} \mathrm{L}^{-1}$ ) sacarosa $(30,000)$, myo-inositol (100), tiamina $\mathrm{HCl}(0.4)$ y TC-agar $\left(\operatorname{Sigma}{ }^{\circledR}\right)$. El pH del medio fue ajustado a 5.7-5.8 previo a la adición del agar y esterilizado a $121{ }^{\circ} \mathrm{C}$ y $1.1 \mathrm{~kg} \mathrm{~cm}^{-2}$ por 15 minutos. Con el fin de determinar el mejor tratamiento para la inducción de callo morfogénico, se evaluó el efecto de diferentes concentraciones $\left(0.0,0.5,1.0,2.0\right.$ y $\left.4.0 \mathrm{mg} \mathrm{L}^{-1}\right)$ de bencilaminopurina (BAP) combinadas con tres concentraciones $\left(0.0,0.5\right.$ y $\left.1.0 \mathrm{mg} \mathrm{L}^{-1}\right) \mathrm{de}$ ácido naftalenacético (ANA) para un total de 15 tratamientos. Aproximadamente $30 \mathrm{ml}$ de medio, independientemente suplementado con los tratamientos mencionados, fueron vertidos en cada caja de Petri $^{\circledR}$; un explante fue establecido por cada recipiente, los cuales fueron sellado con Parafilm ${ }^{\circledR}$ y almacenados en completa oscuridad durante cuatro semanas a una temperatura de $25{ }^{\circ} \mathrm{C}$, al final de las cuales se evaluaron las variables de porcentaje de inducción de callo, friabilidad y presencia o ausencia de órganos (tallos y raíces). Seguidamente, para determinar el efecto de las concentraciones de BAP en combinación con ANA sobre el incremento de masa fresca de callo, los tejidos desarrollados en los tratamientos que indujeron los mayores niveles de formación de callo friable fueron seleccionados y transferidos a medio fresco con la misma formulación y concentración de reguladores de crecimiento de la etapa de inducción. Cada tratamiento tuvo 20 repeticiones distribuidas completamente al azar, donde una repetición consistió de una muestra de tejido en una caja de Petri, registrándose el peso de cada una de las muestras establecidas. Los cultivos fueron almacenados en condiciones similares a las de la etapa de inducción por un período de 4 semanas, luego de las cuales se realizó un nuevo pesaje, registrándose la diferencia de pesos con relación al peso inicial. Los datos registrados fueron analizados con una análisis de varianza con base en el modelo estadístico $\mathrm{Y}_{i j k}=\mathrm{m}+\mathrm{a}_{i}+\mathrm{b}_{j}+(\mathrm{ab})_{i j}+\varepsilon_{i j k j}$ donde $\mathrm{m}$ es el promedio general, $a_{i}$ representa el efecto de las concentraciones de $\mathrm{BAP}, \mathrm{b}_{j}$ representa el efecto de las concentraciones de ANA, $(a b)_{i j}$ representa la interacción de BAP con ANA y $\varepsilon_{i j k}$ es el componente del error al azar.

\section{Regeneración de plantas}

Una vez determinado el mejor tratamiento para incrementar la masa fresca de los tejidos, el callo producido fue proliferado y después de un subcultivo (cuatro semanas después de establecido) transferido a medios semisólidos con formulaciones similares a la fase de establecimiento pero suplementados independientemente con diferentes niveles (0.0, 0.25, 0.5, 1.0, 2.0 y $\left.4.0 \mathrm{mg} \mathrm{L}^{-1}\right)$ de BAP. Además se utilizaron las siguientes combinaciones de ANA/BAP (en mg L $\mathrm{L}^{-1}$ ) 0.5/ $2.0,0.5 / 4.0,1.0 / 2.0$ y 1.0/4.0, con el fin de evaluar su efecto sobre la regeneración de tallos. Aproximadamente $100 \mathrm{mg}$ de tejido de callo fueron inoculados sobre medios de cultivo y almacenados a una temperatura aproximada de $25{ }^{\circ} \mathrm{C}$ en presencia de luz blanca fluorescente $\left(40 \mu \mathrm{mol} \mathrm{m} \mathrm{m}^{-2} \mathrm{~s}^{-1}\right)$. Al final de la octava semana de cultivo se evaluó en cada tratamiento el número de órganos desarrollados con el fin de determinar el mejor tratamiento de regeneración.

\section{RESULTADOS Y DISCUSIÓN}

\section{Inducción y multiplicación de callo}

Los datos registrados permitieron observar que la presencia de auxina en el medio de cultivo es necesaria y suficiente para inducir la formación de callos en los explantes de Stevia. La figura 1 muestra que al menos el $80 \%$ de los explantes cultivados desarrollaron callos en presencia de ANA o ANA + BAP, mientras 
que menos de un $5 \%$ de los explantes cultivados en la dosis máxima de BAP (4 mg $\mathrm{L}^{-1}$ ) desarrollaron algún tipo de cultivo. Tisserat (1985), demostró que la producción de callos está íntimamente ligada a la adición de auxinas al medio de cultivo, y que este efecto podría verse potencializado por la adición conjunta de auxinas y citocininas en el mismo medio de cultivo. Solange et al. (2002), encontraron que la ausencia de reguladores de crecimiento en el medio de cultivo evitaba la formación de callos en Tridax procumbens, comparado con medios suplementados con varias auxinas (2,4-D; IBA y ANA) en presencia de BAP, y observando que el mejor tratamiento para inducir callos en la especie antes mencionada era la combinación en igual proporción de ANA y BAP (2.0 $\left.\mathrm{mg} \mathrm{L}^{-1}\right)$, lo cual confirma que la combinación de auxinas y citocininas promueve la formación de tejido calloso.

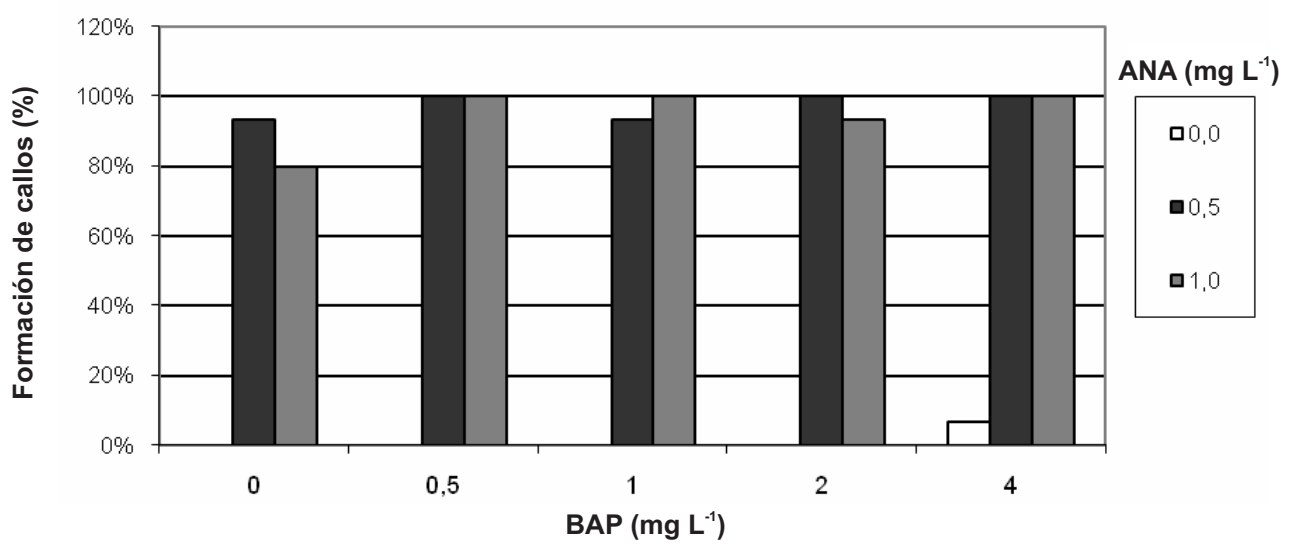

Figura 1. Efecto de diferentes concentraciones de ANA y BAP la formación de callos de Stevia rebaudianain vitro.

Los porcentajes de friabilidad de callo oscilaron entre el $7 \%$ y el $60 \%$ (Figura 2, Figura 3a). El mayor porcentaje de friabilidad se obtuvo en el tratamiento con combinaciones $0.5 \mathrm{mg} \mathrm{L}^{-1}$ de ANA $+4.0 \mathrm{mg}$ $\mathrm{L}^{-1}$ de $\mathrm{BAP}$, mientras que los valores mas bajos en esta variable ocurrieron cuando el medio estaba desprovisto de BAP. Los bajos porcentajes de friabilidad en presencia de ANA solamente indica la necesidad de la interacción de ambas hormonas en el medio para obtener tejidos friables. Sánchez et al. (2005) evaluaron el efecto de auxinas y citocininas en la producción de callo en explantes de Tropaeolum tuberosum (Tropaeolaceae), obteniendo únicamente callos compactos en tratamientos donde se combinaron diferentes concentraciones de ANA con $0.5 \mathrm{mg} \mathrm{L}^{-1}$ de BAP.
La formación de brotes caulinares bien diferenciados y desarrollados con morfologías normales ocurrió en varios de los cultivos en presencia de diferentes tratamientos, observándose la mayor formación cuando los explantes fueron cultivados en presencia de $1.0 \mathrm{mg} \mathrm{L}^{-1}$ de ANA + $4.0 \mathrm{mg} \mathrm{L}^{-1}$ de BAP (Tabla 1). Los brotes mostraron una coloración inicial amarillo-blanquecino producto de la oscuridad aunque rápidamente se tornaron de color verde ante la presencia de luz (Figura 3). De forma alterna, se observó el desarrollo de estructuras radicales notándose un mayor número de estas cuando los explantes fueron cultivados en medios suplementados solo con ANA (Tabla 2; Figura 3). Las variaciones en la formación de órganos en los diferentes tratamientos no permitió establecer una correlación directa entre la formación de 


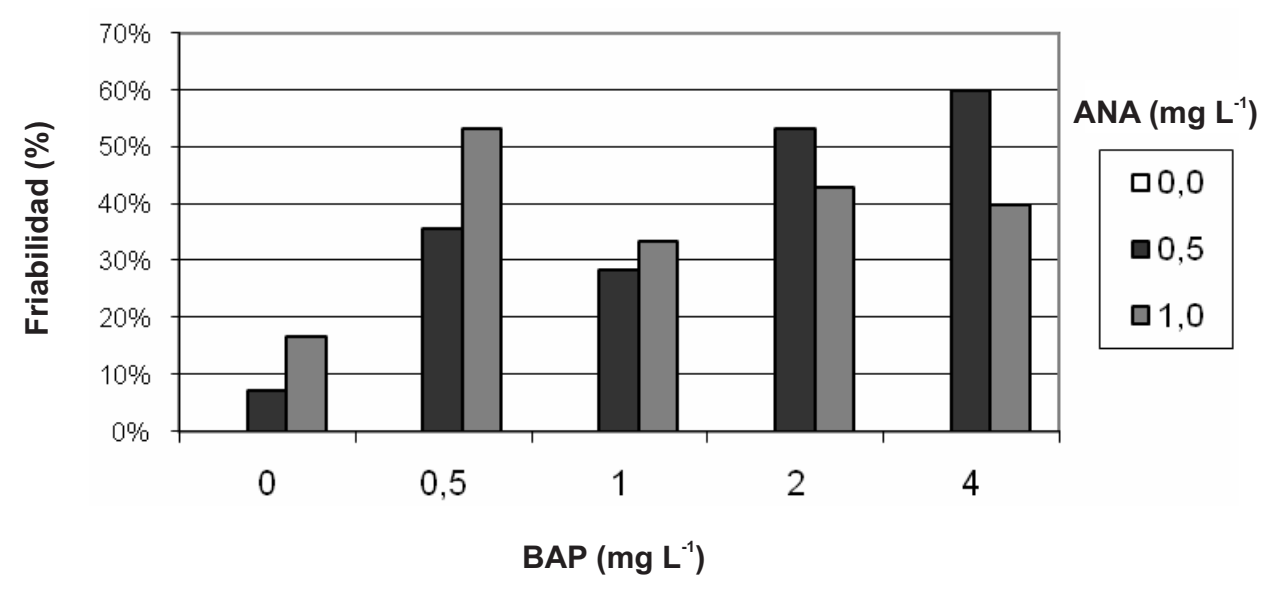

Figura 2. Efecto de diferentes concentraciones de ANA y BAP la formación de callos friables de Stevia rebaudiana.
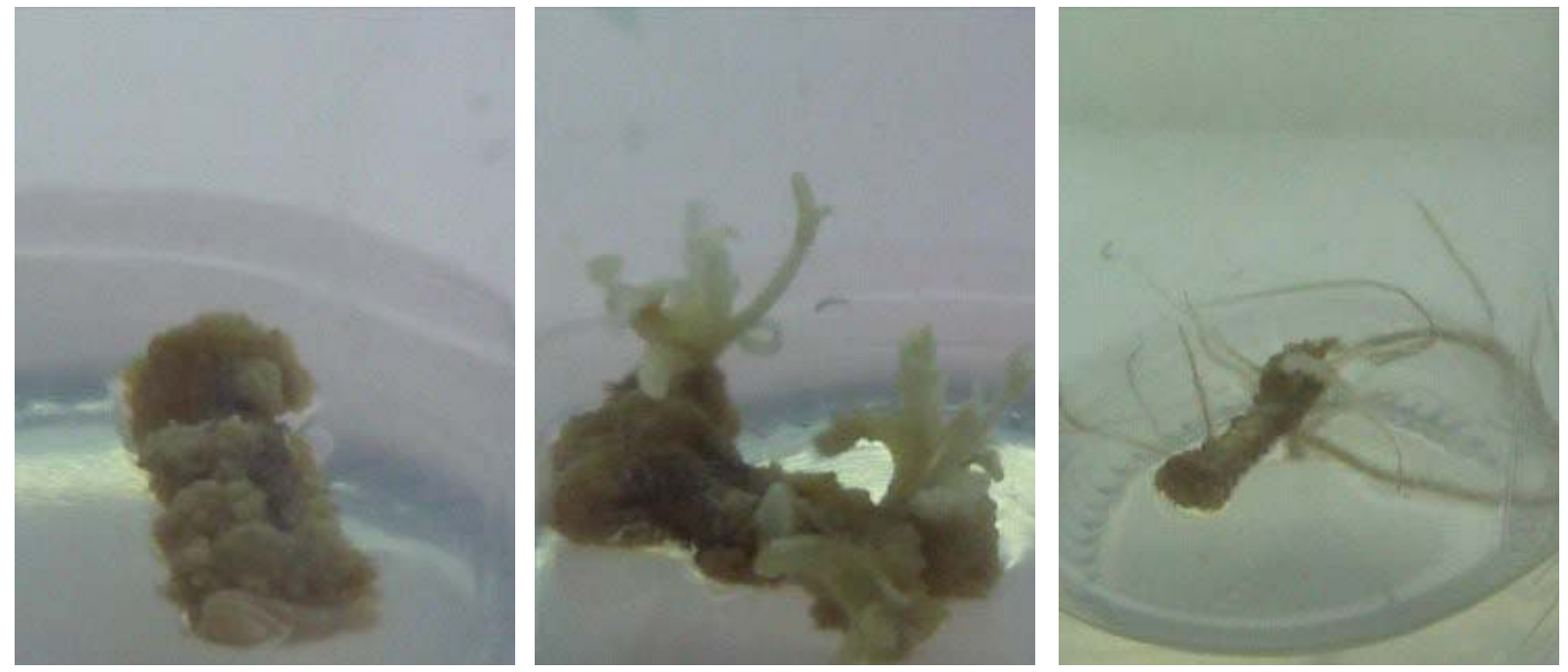

Figura 3. Orgaonogénesis de Stevia rebaudiana Bertoni; Callo (izquierda), regeneración de tallos (centro), regeneración de raíces (derecha).

órganos con los tratamientos evaluados. Litz et al. (1983) afirman que la concentración de reguladores de crecimiento requeridos para la organogénesis puede variar de un tejido a otro dentro de la misma planta, o entre plantas de la misma especie, lo cual supone que la respuesta de un determinado explante puede variar de la respuesta de otro explante bajo las mismas concentraciones de hormonas. Flick et al. (1983) reportaron que algunas veces puede darese la ocurrencia de organogénesis directa en explantes cultivados en medios que contengan únicamente citocinina ya que esta depende de los niveles endógenos de reguladores de crecimiento vegetal en el momento de la organogénesis. Capote et al. (2000) reconocieron la capacidad de ANA en la formación de raíces en callos inducidos a partir de explantes de cebolla 
Tabla 1. Efecto de diferentes concentraciones de ANA y BAP el número de tallos y raíces (tallos/raíces) de Stevia rebaudiana regenerados in vitro.

\begin{tabular}{ccccccc}
\hline & & \multicolumn{5}{c}{ BAP $\left(\mathbf{m g ~ L}^{-1}\right)$} \\
\cline { 2 - 6 } & & $\mathbf{0}$ & $\mathbf{0 , 5}$ & $\mathbf{1}$ & $\mathbf{2}$ & $\mathbf{4}$ \\
\hline \multirow{3}{*}{ ANA $\left(\mathbf{m g ~ L}^{-1}\right)$} & $\mathbf{0}$ & $1 / 4$ & $0 / 1$ & $0 / 1$ & $0 / 3$ & $0 / 0$ \\
& $\mathbf{0 , 5}$ & $1 / 44$ & $3 / 1$ & $0 / 2$ & $3 / 0$ & $3 / 2$ \\
& $\mathbf{1}$ & $0 / 40$ & $0 / 0$ & $1 / 2$ & $0 / 0$ & $9 / 0$ \\
\hline
\end{tabular}

(Allium cepa) y admiten que este evento morfogenético es indeseable en la etapa de inducción de callo, debido a que afecta el crecimiento óptimo del mismo. Matos et al. (1995), obtuvieron callos y raíces en explantes foliares de Capraria biflora (Scrophulariaceae) al ser establecidos sobre medios de cultivo suplementados con ANA $\left(1.0 \mathrm{mg} \mathrm{L}^{-1}\right)$ tanto en condiciones de luz como en oscuridad; en un segundo experimento, los mismos autores evaluaron la misma concentración de ANA combinada con kinetina ( $\left.0.1 \mathrm{mg} \mathrm{L}^{-1}\right)$ observando solo la formación de callos; sin embargo, cuando se combinaron $1.0 \mathrm{mg} \mathrm{L}^{-1}$ ANA con $1.0 \mathrm{mg}$ $\mathrm{L}^{-1}$ BAP hubo formación tanto de callos solamente como de callos, tallos y raíces en forma simultanea. Estos resultados respaldan los datos obtenidos en el presente trabajo, comprobándose la influencia del ANA, solo y en combinación con citocininas en la inducción de callo y la formación de órganos adventicios, respectivamente.

Los resultados del análisis de varianza no permitieron detectar diferencias estadísticamente significativas ( $P r>0.05)$; con respecto al incremento de masa fresca en las combinaciones seleccionadas; no obstante, los resultados obtenidos permitieron observar que el tratamiento con $0.5 \mathrm{mg} \mathrm{L}^{-1} \mathrm{ANA}+1.0 \mathrm{mg} \mathrm{L}^{-1}$ BAP indujo el mayor incremento de masa fresca (313.4 $\mathrm{mg}$ ), mientras que el tratamiento con $1.0 \mathrm{mg}$ $\mathrm{L}^{-1} \mathrm{ANA}+0.5 \mathrm{mg} \mathrm{L}^{-1}$ BAP reportó el menor crecimiento (174.9 mg); los tratamientos con $0.5 \mathrm{mg} \mathrm{L}^{-1} \mathrm{ANA}+2.0 \mathrm{mg} \mathrm{L}^{-1}$ BAP y $1.0 \mathrm{mg} \mathrm{L}^{-1}$
$\mathrm{ANA}+4.0 \mathrm{mg} \mathrm{L}^{-1} \mathrm{BAP}$ reportaron incrementos de $232.4 \mathrm{mg}$ y $176.7 \mathrm{mg}$, respectivamente. Las combinaciones de auxinas y citocininas han demostrado contribuir con la proliferación in vitro de tejidos de callo en algunas especies. Montoya (1991), explica que además de los tipos de reguladores de crecimiento y las cantidades en que son suplementadas en el medio son las interacciones cuantitativas entre los reguladores de crecimiento presentes, las que mayoritariamente proporcionan el mecanismo de regulación de los fenómenos morfogenéticos durante el cultivo in vitro. Por su parte Villalobos y Thorpe (1991) argumentan que la diferenciación de novo está ligada a la proliferación celular y la organización de las nuevas células sigue una "programación" influenciada por las condiciones de cultivo in vitro conjuntamente con la ganancia de peso. Solange et al. (2002) no solo encontraron que la combinación de $2.0 \mathrm{mg}$ $\mathrm{L}^{-1}$ de ANA + $2.0 \mathrm{mg} \mathrm{L}^{-1}$ de BAP promovía la formación de callos, sino también que la misma combinación favorecía el incremento de masa fresca de callos en Tridax procumbens (Asteraceae), y que a medida que se incrementaban las concentraciones de auxina, decrecía el peso fresco de los callos; lo cual presenta cierta concordancia con los resultados obtenidos en este trabajo desde el punto de vista de la relación entre los reguladores de crecimiento, y el incremento de masa fresca de los callos proliferados. Por su parte, Siddique et al. (2003) obtuvieron resultados semejantes al 
inducir callos en explantes caulinares de Hemidesmus indicus (Asclepiadaceae), encontrando que la combinación de $1.0 \mathrm{mg}$ $\mathrm{L}^{-1}$ de $\mathrm{ANA}+2.0 \mathrm{mg} \mathrm{L}^{-1}$ de kinetina favoreció considerablemente el incremento de peso fresco.

\section{Regeneración de Plantas}

Los tratamientos aplicados no indujeron la regeneración de plantas de Stevia a partir de los tejidos de callo proliferados en condiciones in vitro. Los resultados obtenidos en la fase de inducción, donde se observó la regeneración de órganos, indican que los tejidos tenían potencial morfogenético suficiente para llevar a cabo la organogénesis, y que la ausencia de respuesta a la formación de estructuras a partir del callo en la etapa final es posiblemente una consecuencia de la disminución de este potencial como resultado de los continuos subcultivos realizados o la necesidad de seguir experimentando hasta encontrar el ajuste adecuado en el medio de cultivo que suministre las necesidades para la regeneración (Christianson y Warnik, 1985). La presencia de citocininas en el medio de cultivo no garantiza la respuesta del callo hacia la formación del brote. Krikorian (1991) reconoce que a pesar que en muchas especies vegetales puede inducirse la proliferación de tejido calloso y posteriormente la formación de brotes y raíces manipulando la relación auxina/citocinina aplicada exógenamente, no pueden universalizarse dichos acontecimientos, especialmente en cuanto a la respuesta morfogenética del callo como consecuencia de la adición exógena de citocininas. La ausencia de organogénesis a partir de callo es frecuente en varias especies, especialmente en aquellas consideradas recalcitrantes para el cultivo in vitro (Yaya et al., 2005). Algunos estudios señalan un efecto inhibitorio del explante sobre la ocurrencia de organogénesis cuando el callo originado se mantiene adherido a este (Litz y Jarret, 1991); sin embargo, los resultados obtenidos en el presente estudio contradicen la anterior premisa puesto que la formación de los órganos regenerados en condiciones in vitro ocurrió en los estados iniciales del proceso cuando el tejido de callo desarrollado y el explante se encontraban unidos en una misma estructura. Adicionalmente, la separación del explante inicial del tejido calloso durante la etapa de multiplicación y posterior intento de regeneración no contribuyeron con la formación de órganos. Es posible que el tipo de explante utilizado tenga un efecto sobre los resultados obtenidos ya que existen reportes que sugieren la posibilidad de lograr organogénesis en estevia utilizando tejidos foliares como explante inicial (Sivaram y Mukundan, 2003) por lo cual es necesario continuar las investigaciones teniendo en cuenta estos resultados.

\section{CONCLUSIONES}

- La formación de nuevos brotes de Stevia in vitro vía organogénesis es posible en los estados iniciales del proceso.

- La mayor regeneración de brotes ocurrió en la etapa de inducción de callo en presencia de $1.0 \mathrm{mg} \mathrm{L}^{-1}$ de ANA + $4.0 \mathrm{mg} \mathrm{L}^{-1}$ de BAP.

- El suplemento de ácido naftalenacético (ANA) en el medio de cultivo es necesario y suficiente para inducir callo a partir de explantes consistentes de entrenudos de Stevia.

- La combinación de $0.5 \mathrm{mg} \mathrm{L}^{-1}$ de ANA y $1.0 \mathrm{mg} \mathrm{L}^{-1}$ de BAP indujo la mayor multiplicación en callos de Stevia.

- La presencia de BAP en el medio de cultivo no tuvo ningún efecto sobre la regeneración de brotes a partir de callos multiplicados de Stevia en condiciones in vitro. 


\section{BIBLIOGRAFÍA}

Bespalhok, J. y Hattori, K. 1997. Embryogenic callus formation and histological studies from Stevia rebaudiana Bertoni floret explants. Revista Brasileira de Fisiología Vegetal 9(3):185-188

Bespalhok, J.; Hashimoto, J. y Esteves, L. 1993. Induction of somatic embryogenesis from leaf explants of Stevia rebaudiana. Revista Brasileira de Fisiología Vegetal 5(1):51-53

Capote, A.; Fundora, Z. y Pérez, O. 2000. Estudio de la variabilidad inducida en células y plántulas de cebolla (Allium сера, L.) cv. Caribe-71 regeneradas in vitro. Biotecnología Aplicada 17:241-246

Cassacia, J. y Alvarez, E. 2006. Recomendaciones técnicas para una producción sustentable del ka'a he'e (Stevia rebaudiana (Bertoni)) en el Paraguay. Ministerio de Agricultura y Ganadería - Paraguay, Manual Técnico №8, 51 p.

Christianson, M. y Warnik, D. 1985. Temporal requirements for phytohormone balance in the control of organogenesis in vitro. Developmental Biology 112:494

Flick, C.; Evans, D. y Sharp, W. 1983. Organogénesis. En: Evans, D.; Sharp, W. y Ammirato, P. (Ed). Handbook of Plant Cell Culture. MacMillan Publishing, Nueva Cork, p13-81

Krikorian, A. 1991. Propagación clonal in vitro. En: Roca, W. y Mroginski, L. (Ed). Cultivo de Tejidos en la Agricultura: Fundamentos y Aplicaciones. CIAT, Cali, p95-125
Litz, R.; O'Hair, S. y Conover, R. 1983. In vitro growth of Carica papaya L. cotyledons. Scientia Horticulturae 19:287-293

Litz, R. y Jarret, R. 1991. Regeneración de plantas en el cultivo de tejidos: Embriogénesis somática y organogénesis. En: Roca, W. y Mroginski, L. (Ed). Cultivo de Tejidos en la Agricultura: Fundamentos y Aplicaciones. CIAT, Cali, p 143-171

Matos, A.; Da Costa, M. y Handro, W. 1995. Morphogenesis and plant regeneration in leaf explants and callus tissues of Capraria biflora cultured in vitro. Revista Brasileira de Fisiología Vegetal 7(2):171-174

Megeji, N.; Kumar, J.; Singh, V.; Kaul, V. y Ahuja, P. 2005. Introducing Stevia rebaudiana, a natural zero-calorie sweetener. Current Science 88(5):801- 804

Montoya, L. 1991. Cultivo de Tejidos Vegetales. Lealon, Medellín, 77p.

Mroginski, L. y Roca W. 1991. Establecimiento de cultivos de tejidos vegetales in vitro. En Roca, W. y Mroginski L. (Ed.). Cultivo de Tejidos en la Agricultura: Fundamentos y Aplicaciones. CIAT, Cali, P 19-40

Murashige, T. y Skoog, F. 1962. A revised medium for rapid growth and bioassays with tobacco tissue cultures. Physiologia Plantarum 15:473-497

Sánchez, D.; Huamaní, K.; Pascual, E.; Sánchez, H. y Estrada, R. 2005. Efecto 
de las auxinas y citoquininas en la callogénesis sobre explantes nodales e internodales de Tropaeolum tuberosum (r. and p.) mashua. Memorias XIV Reunión científica ICBAR, Lima

Schwarz, O. y Beaty, R. 1996. propagation from non-meristematic tissues organogenesis. En: Trigiano, R. y Gray, D. (Ed). Plant Tissue Cultures Concepts and Laboratory Excercises. CRC Press, Boca Raton, 95-104

Siddique, N.; Bari, M.; Khatun, N.; Rahman, M.; Rahman M. y Huda, S. 2003. Plant regeneration from nodal segments derived callus in Hemidesmus indicus (L.) R. Br (Anantamul) an endangered medicinal plant in Bangladesh. Journal of Biological Sciences 3(12):1158-1163

Sivaram, L. y Mukundan, U. 2003. In vitro culture studies on Stevia rebaudiana. In Vitro Cellular and Developmental Biology-Plant 39:520-523

Solange, E.; Pereira, J.; Ramalho, A.; Arbex, N.; Cardoso, M. y Alves, O. 2002. Indução de calos em erva-de-touro (Tridax procumbens I.) utilizando diferentes reguladores de crescimento e tipos de explantes. Ciencia e Agrotecnologia 26(2):301-308
Soto, A. y Del Val, S. 2002. Extracción de los principios edulcorantes de la Stevia rebaudiana. Revista de Ciencias Agrarias y Tecnología de los Alimentos 20:5-9

Taiariol, D. 1995. Propagación vegetativa de Stevia rebaudiana Bertoni. Tesis Ingeniero Agrónomo, Universidad de Buenos Aires, Buenos Aires.

Tisserat, B. 1985. Embryogenesis, organogenesis and plant regeneration. En: Ixon, R. (Ed). Plant Cell Culture, A Practical Approach. IRL Press, Oxford, p79-105

Villalobos, V. y Thorpe, T. 1991. Micropropagación: conceptos, metodología y resultados. En: Roca, W. y Mroginski, L. (Ed). Cultivo de Tejidos en la Agricultura: Fundamentos y Aplicaciones. CIAT, Cali, p 128-141

Yaya M, Rodríguez O, Usaquén W y Chaparro W. 2005. Inducción de organogénesis directa en abarco (Cariniana pyriformis Miers.). Agronomía Colombiana 23(1):50-54 